\title{
ARTICLE
}

Received 30 Nov 2013 | Accepted 8 Apr 2014 | Published 30 Apr $2014 \quad$ DOI: 10.1038/ncomms4825

\section{Tuning the surface Fermi level on p-type gallium nitride nanowires for efficient overall water splitting}

\author{
M.G. Kibria', S. Zhao ${ }^{1}$, F.A. Chowdhury ${ }^{1}$, Q. Wang${ }^{1}$, H.P.T. Nguyen ${ }^{1}$, M.L. Trudeau², H. Guo ${ }^{3}$ \& Z. Mi ${ }^{1}$
}

Solar water splitting is one of the key steps in artificial photosynthesis for future carbonneutral, storable and sustainable source of energy. Here we show that one of the major obstacles for achieving efficient and stable overall water splitting over the emerging nanostructured photocatalyst is directly related to the uncontrolled surface charge properties. By tuning the Fermi level on the nonpolar surfaces of gallium nitride nanowire arrays, we demonstrate that the quantum efficiency can be enhanced by more than two orders of magnitude. The internal quantum efficiency and activity on $p$-type gallium nitride nanowires can reach $\sim 51 \%$ and $\sim 4.0$ mol hydrogen $\mathrm{h}^{-1} \mathrm{~g}^{-1}$, respectively. The nanowires remain virtually unchanged after over $50,000 \mu \mathrm{mol}$ gas (hydrogen and oxygen) is produced, which is more than 10,000 times the amount of photocatalyst itself ( $\sim 4.6 \mu \mathrm{mol})$. The essential role of Fermi-level tuning in balancing redox reactions and in enhancing the efficiency and stability is also elucidated.

\footnotetext{
${ }^{1}$ Department of Electrical and Computer Engineering, McGill University, 3480 University Street, Montreal, Québec, Canada H3A 0E9. ${ }^{2}$ Science des Matériaux, IREQ, Hydro-Québec, 1800 Boulevard Lionel-Boulet, Varennes, Québec, Canada J3X1S1. ${ }^{3}$ Centre for the Physics of Materials, Department of Physics, McGill University, 3600 University Street, Montreal, Québec, Canada H3A 2T8. Correspondence and requests for materials should be addressed to Z.M. (email: zetian.mi@mcgill.ca).
} 
A mong the various paths to solar fuel production, the photochemical dissociation of water into its constituent parts, $\mathrm{H}_{2}$ and $\mathrm{O}_{2}$, offers the simplest and potentially the most efficient approach; this spontaneous, wireless approach requires virtually zero energy input except sunlight ${ }^{1-7}$. Recently, nanoscale photocatalysts have been intensively studied that can increase light absorption and charge carrier separation, and therefore enhance the quantum efficiency ${ }^{8-10}$. Fermi-level pinning, however, has been commonly measured on nanowire surfaces ${ }^{11}$. The resulting surface band bending creates an additional energy barrier for charge carrier transport to the photocatalyst-water interface ${ }^{12}$, leading to significantly reduced reaction rate and extremely low efficiency ${ }^{13,14}$. To date, the rational synthesis of nanostructured photocatalysts with controlled surface charge properties, that is, tunable surface Fermi level and band bending, has remained a near-universal challenge ${ }^{9}$. Such uncontrolled surface charge properties can further contribute to the photocorrosion and instability of various nanostructures under harsh photocatalysis conditions, severely limiting their practical applications.

In this study, we examine the water splitting reaction on the nonpolar surfaces ( $m$-plane) of $\mathrm{GaN}$ nanowire photocatalyst, which may further serve as a new platform for spontaneous water splitting under visible and infrared light irradiation by incorporating indium ${ }^{15,16}$. Unique to the nonpolar GaN surfaces is that the occupied surface states are positioned outside of the fundamental energy bandgap and therefore do not create Fermi-level pinning ${ }^{17,18}$. The nonpolar surfaces are also highly reactive for spontaneous dissociation of water molecules and possess low energy barrier for proton diffusion ${ }^{19,20}$. Owing to the impurity incorporation and/or the presence of any defects, however, an upward (downward) band bending has been commonly measured on $n$ - $(p-)$ type GaN surfaces ${ }^{21,22}$. When $n$-type GaN surfaces come into contact with water, the upward band bending becomes more severe, as shown in Fig. 1a,b, which can suppress $\mathrm{H}_{2} \mathrm{O}$ reduction reaction. When $p$-type $\mathrm{GaN}$ surfaces are in equilibrium with water, the downward band bending can be enhanced, as shown in Fig. 1c,d, which can limit the $\mathrm{H}_{2} \mathrm{O}$ oxidation reaction. In practice, weakly $n$-type or nearly intrinsic surfaces are often present on $p$-doped $\mathrm{GaN}$ nanowires, with the Fermi level positioned slightly above the electrochemical potential of water. As a consequence, the downward band bending may actually be reduced when such $p$-doped $\mathrm{GaN}$ nanowires are in equilibrium with water. This provides a distinct opportunity to potentially realize balanced and therefore more efficient redox reactions through tuning the Fermi level on nanowire surfaces. Since water oxidation is often the rate-limiting reaction ${ }^{20,23}$, $p$-doped nanowire photocatalyst can further enhance the overall water splitting efficiency by providing abundant-free holes. To date, however, the achievement of $p$-doped $\mathrm{GaN}$ nanowires with tunable surface Fermi level has remained challenging 24,25 (Supplementary Note 1). In this study, we demonstrate that the near-surface band bending of catalyst-free $\mathrm{GaN}$ nanowires can be controlled with $p$-type $\mathrm{Mg}$-dopant incorporation. With optimized surface charge properties, we show that the efficiency of spontaneous overall water splitting may be enhanced by more than two orders of magnitude.

\section{Results}

Structural characterization. The GaN nanowires were grown on Si substrate by plasma-assisted molecular beam epitaxy (MBE; Methods section). The Mg-dopant concentration was varied by tuning the $\mathrm{Mg}$ effusion cell temperatures $\left(T_{\mathrm{Mg}}\right)$ from 200 to $300^{\circ} \mathrm{C}$. Samples A, B, C, D, E and $\mathrm{F}$ correspond to $T_{\mathrm{Mg}}$ of $200^{\circ} \mathrm{C}, 230^{\circ} \mathrm{C}, 250^{\circ} \mathrm{C}, 265^{\circ} \mathrm{C}, 280^{\circ} \mathrm{C}$ and $300^{\circ} \mathrm{C}$, respectively.
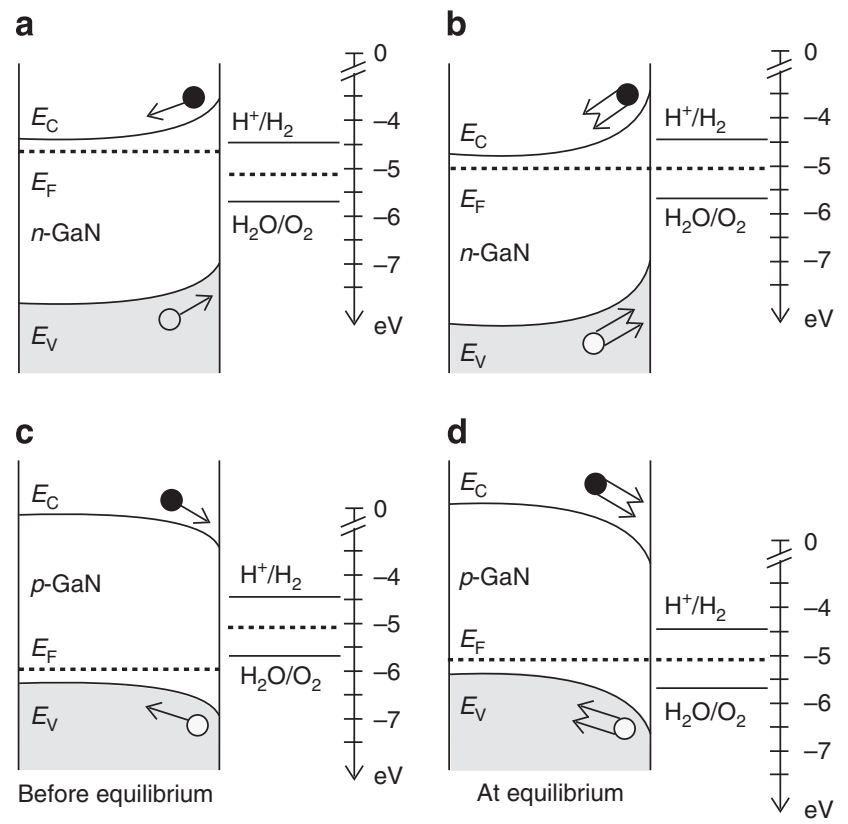

Figure 1 | Surface band bending of $n$ - and $p$-type GaN nanowires.

Schematic illustration of the energy band diagram of $n$-type GaN nanowire surface (a) before and (b) at equilibrium with water. The upward band bending becomes more severe at equilibrium, which suppresses water reduction reaction. Schematic illustration of the energy band diagram of p-type GaN nanowire surface (c) before and (d) at equilibrium with water. The downward band bending gets enhanced at equilibrium that suppresses water oxidation reaction.

A $45^{\circ}$-tilted scanning electron microscopy (SEM) image of asgrown $\mathrm{Mg}$-doped GaN (denoted as GaN:Mg hereafter) nanowire (sample B) is shown in Fig. 2a (also Supplementary Fig. 1 for SEM images of other samples). The nanowires are $\sim 600 \mathrm{~nm}$ in length, $\sim 50-75 \mathrm{~nm}$ in diameter and vertically aligned to the substrate. Shown in Fig. $2 \mathrm{~b}$ is a scanning transmission electron microscopy bright-field (STEM-BF) image, illustrating lattice fringes from defect-free single crystalline nanowire (Methods section). The distance between the two adjacent fringes is about $\sim 0.518 \mathrm{~nm}$ that corresponds to the $<0001>$ direction, further confirming that the nanowires are grown along the $c$ axis, with their sidewalls being nonpolar $m$-planes ${ }^{26}$. Room temperature photoluminescence (PL) measurement clearly shows a single optical emission peak at $\sim 365 \mathrm{~nm}$, corresponding to the bandgap of $\mathrm{GaN}$ (3.4 eV) (Methods section and Supplementary Fig. 2). For subsequent experiments $\sim 2.8 \mathrm{~cm}^{2}$ wafer sample was used that corresponds to $\sim 0.387 \mathrm{mg}(\sim 4.62 \mu \mathrm{mol}) \mathrm{GaN}$ materials, considering the nanowire fill factors on $\mathrm{Si}$ substrate (Supplementary Fig. 3).

Near-surface band structure. The near-surface band structure of as-grown nanowires can be estimated by measuring the Fermi level $\left(E_{\mathrm{F}}\right)$ relative to the valence band maximum $\left(E_{\mathrm{V}}\right)$. The $E_{\mathrm{F}}-E_{\mathrm{V}}$ in the near-surface region (Fig. 2c inset) was measured by recording angle-resolved X-ray photoelectron spectroscopy (ARXPS) valence spectrum (Fig. 2c inset) from the lateral nonpolar ( $m$-plane) surfaces of the nanowire (Methods section) ${ }^{27}$. Illustrated in Fig. 2c, the $E_{\mathrm{F}}-E_{\mathrm{V}}$ is measured to be $2.63 \mathrm{eV}$ for undoped $\mathrm{GaN}\left(T_{\mathrm{Mg}}=0{ }^{\circ} \mathrm{C}\right)$, which is similar to the $E_{\mathrm{F}}-E_{\mathrm{V}}$ $(2.6 \mathrm{eV})$ measured on $n$-type GaN surfaces by Wu et al. ${ }^{28}$ Two distinct regimes are clearly separated with dotted lines. Regime I (n-type surfaces), wherein the near-surface $E_{\mathrm{F}}-E_{\mathrm{V}}$ stays nearly 
a

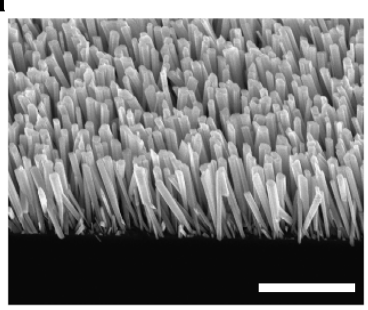

b

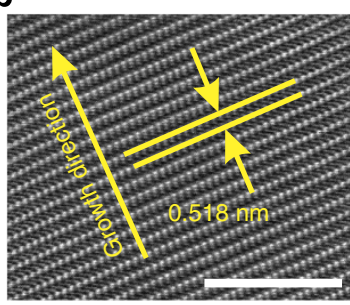

d

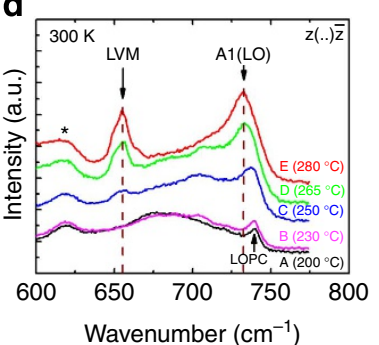

e

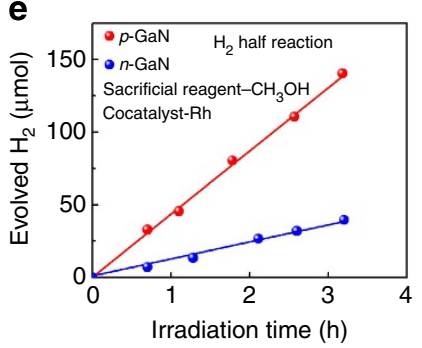

c

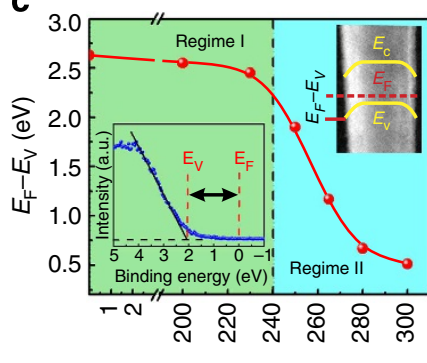

Mg cell temperature $\left({ }^{\circ} \mathrm{C}\right)$

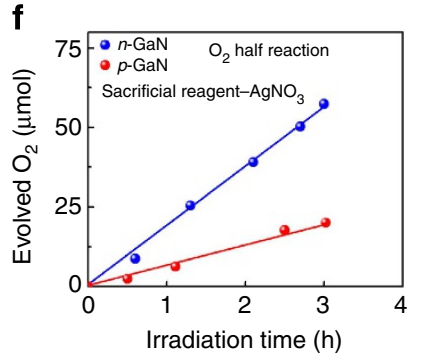

Figure 2 | Structural characterization and surface charge properties of GaN:Mg nanowires. (a) A $45^{\circ}$ tilted SEM image of GaN:Mg (sample B) nanowire arrays. Scale bar, $1 \mu \mathrm{m}$. (b) STEM-BF lattice image illustrating lattice fringes from defect-free single crystalline GaN:Mg nanowire (sample B). Scale bar, $5 \mathrm{~nm}$. (c) $E_{\mathrm{F}}-E_{\mathrm{V}}$ for different $\mathrm{GaN}: M g$ samples determined from ARXPS valence spectrum. The insets illustrate $E_{\mathrm{F}}-E_{\mathrm{V}}$ on the TEM image of a single nanowire and on an ARXPS valence spectrum. The dotted line separates two regimes, Regime I ( $n$-type surfaces) and Regime II ( $p$-type surfaces). (d) Typical room temperature micro-Raman spectra of $\mathrm{GaN}$ nanowires with $T_{\mathrm{Mg}}$ ranging from 200 to $280^{\circ} \mathrm{C}$. Si modes are pointed out with '*' and the dashed lines are guide to the eye. (e) Evolution of $\mathrm{H}_{2}$ from $n$ - (Si-doped) and $p$ - $\mathrm{GaN}\left(\mathrm{T}_{\mathrm{Mg}}=230^{\circ} \mathrm{C}\right.$ ) nanowire arrays in the presence of $\mathrm{CH}_{3} \mathrm{OH}$ and Rh cocatalyst. Enhanced evolution of $\mathrm{H}_{2}$ is observed for $p$-type $\mathrm{GaN}$ nanowires, owing to the presence of downward surface band bending. (f) Evolution of $\mathrm{O}_{2}$ from $n$ - (Si-doped) and $p-\mathrm{GaN}\left(\mathrm{T}_{\mathrm{Mg}}=230^{\circ} \mathrm{C}\right.$ ) nanowire arrays in the presence of $\mathrm{AgNO}_{3}$ sacrificial reagent. Enhanced evolution of $\mathrm{O}_{2}$ is measured for $n$-type GaN nanowires, which can be well correlated to the presence of upward surface band bending. The solid lines are guide to the eye.

constant for $T_{\mathrm{Mg}}$ up to $230^{\circ} \mathrm{C}$, which corresponds to the compensation of background $n$-type dopants by $\mathrm{Mg}$ acceptors ${ }^{29}$. Regime II ( $p$-type surfaces), wherein the near-surface $E_{\mathrm{F}}-E_{\mathrm{V}}$ decreases drastically with increase in $T_{\mathrm{Mg}}$, is because of the enhancement in free hole concentration in $\mathrm{GaN}$ nanowires. In the relatively high bulk-doping regime (samples $\mathrm{C}-\mathrm{F}$ ), variations of $E_{\mathrm{F}}-E_{\mathrm{V}}$ in the bulk region with increasing $\mathrm{Mg}$ cell temperature is estimated to be in the range of tens of $\mathrm{meV}$, which is much smaller than the variation measured in the near-surface region $(\sim 2 \mathrm{eV})$. Consequently, the reduction of $E_{\mathrm{F}}-E_{\mathrm{V}}$ in the nearsurface region, as shown in Fig. $2 c$, can be directly correlated to the reduction in the downward surface band bending with increasing $\mathrm{Mg}$-dopant incorporation ${ }^{30,31}$. In addition, our detailed analysis reveals that variations in the surface band bending have a very small, or negligible dependence on the morphology of GaN nanowires.

$p$-Type doping. Variations of the surface charge properties are further probed by micro-Raman measurements at room temperature (Methods section). The evolution of the Raman mode in the range of $600-800 \mathrm{~cm}^{-1}$ with $\mathrm{Mg}$ doping is illustrated in Fig. 2d (the complete Raman spectrum is shown in Supplementary Fig. 4). For nanowires with the lowest Mg-doping levels (sample $\mathrm{A}, \mathrm{T}_{\mathrm{Mg}}=200^{\circ} \mathrm{C}$; and sample $\mathrm{B}, \mathrm{T}_{\mathrm{Mg}}=230^{\circ} \mathrm{C}$ ), a narrow peak at $\sim 741 \mathrm{~cm}^{-1}$ is present, which is attributed to the coupling between the longitudinal optical phonon-plasmoncoupled (LOPC) mode 24,32 . The appearance of the LOPC mode is associated with the weakly $n$-type surfaces for $\mathrm{GaN}$ nanowires at relatively low $\mathrm{Mg}$ doping (samples $\mathrm{A}$ and $\mathrm{B}$ ), marked as Regime I in Fig. 2c, which can be gradually compensated with further increase in $\mathrm{Mg}$ doping/free hole concentration within nanowires.
Accordingly, with the increment of Mg-doping level for sample $\mathrm{C}$ $\left(T_{\mathrm{Mg}}=250^{\circ} \mathrm{C}\right)$, the LOPC mode gradually shifts to a lower frequency, and also its intensity becomes stronger because of the reduced coupling effect between the LO phonon and electron plasmon. With further increment of the Mg-doping level in sample $\mathrm{D}\left(T_{\mathrm{Mg}}=265^{\circ} \mathrm{C}\right)$, the frequency of the LOPC mode shifts to $\sim 733 \mathrm{~cm}^{-1}$ (the original frequency of the pure uncoupled $\mathrm{A}_{1}$ (LO) mode). It indicates that the coupling between the LO phonon and electron plasma is completely suppressed in more heavily doped GaN nanowires, which is consistent with the measured surface type (weakly $p$-type, Regime II shown in Fig. 2c). The presence of $p$-type surfaces, evidenced by both the ARXPS and the pure uncoupled $A_{1}(L O)$ mode, is also well correlated with the appearance of an extra mode at $655 \mathrm{~cm}^{-1}$ in sample $\mathrm{C}\left(T_{\mathrm{Mg}}=250^{\circ} \mathrm{C}\right)$. Such a foreign Raman mode is attributed to the local vibrational modes related to $\mathrm{Mg}$ impurity $(\mathrm{Mg}-\mathrm{N} \text { bond })^{33}$. Its intensity is directly proportional to the free hole concentration (Supplementary Fig. 5) ${ }^{28}$ and becomes stronger with further increment of Mg-doping level in samples $\mathrm{D}\left(T_{\mathrm{Mg}}=265^{\circ} \mathrm{C}\right)$ and $\mathrm{E}\left(T_{\mathrm{Mg}}=280^{\circ} \mathrm{C}\right)$.

$\mathbf{H}_{2}$ and $\mathrm{O}_{2}$ half reactions. The tunable surface Fermi level and the engineered surface charge properties are essential to achieve controlled redox reactions. To demonstrate this hypothesis, $\mathrm{H}_{2}$ and $\mathrm{O}_{2}$ half reactions in the presence of respective sacrificial reagents were first performed using both $n$ - (Si-doped) and $p$-type $\mathrm{GaN}$ nanowire arrays (Methods section). Figure 2e shows the evolution of $\mathrm{H}_{2}$ from $n$ - and $p$-type (sample $\mathrm{B}, \mathrm{T}_{\mathrm{Mg}}=230^{\circ} \mathrm{C}$ ) $\mathrm{GaN}$ nanowire arrays in the presence of Methanol $\left(\mathrm{CH}_{3} \mathrm{OH}\right)$ and Rhodium ( $\mathrm{Rh}$ ) cocatalyst. The $\mathrm{H}_{2}$ generation rate of $p$-type $\mathrm{GaN}$ nanowire is significantly higher than conventional n-type GaN 

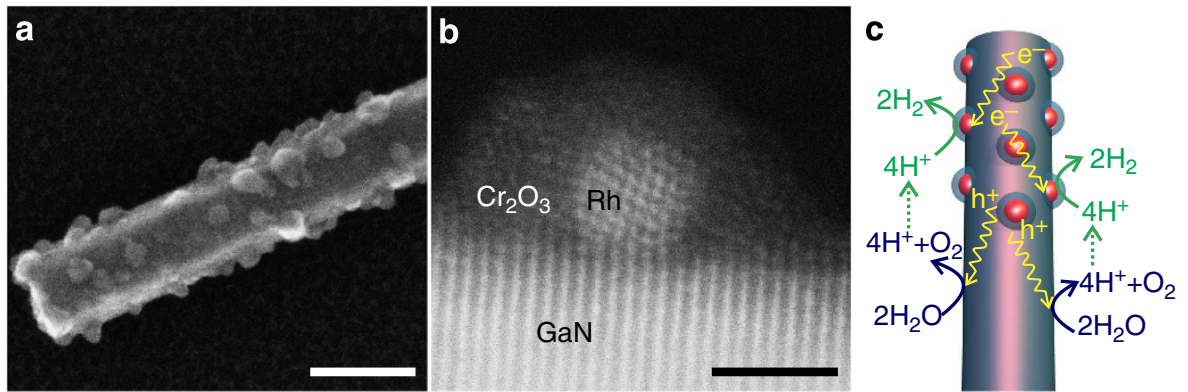

Figure 3 | TEM characterization of core-shell $\mathbf{R h} / \mathbf{C r}_{\mathbf{2}} \mathbf{O}_{\mathbf{3}}$ nanoparticles on $\mathbf{G a N}$ nanowire. (a) Low-magnification STEM-SE image. Scale bar, 50 nm. (b) STEM-HAADF image of $\mathrm{Rh} / \mathrm{Cr}_{2} \mathrm{O}_{3}$ nanoparticle-decorated $\mathrm{GaN}: \mathrm{Mg}$ nanowire. Scale bar, $2 \mathrm{~nm}$. Crystalline $\mathrm{Rh}$ core and amorphous $\mathrm{Cr}_{2} \mathrm{O}_{3}$ shell are clearly observed. (c) Schematic of $\mathrm{Rh} / \mathrm{Cr}_{2} \mathrm{O}_{3}$-decorated $\mathrm{GaN}$ nanowire and the water splitting mechanism.

nanowires, owing to the downward band bending at the surface of p-type GaN (Fig. 1c). In contrast, as shown in Fig. 2f, the activity for $\mathrm{O}_{2}$ generation is significantly enhanced on n-type $\mathrm{GaN}$ nanowires with an upward surface band bending (Fig. 1a). For overall water splitting and stoichiometric evolution of $\mathrm{H}_{2}$ and $\mathrm{O}_{2}$; however, both oxidation and reduction reaction need to proceed simultaneously ${ }^{4}$. The presence of any upward or downward band bending will create additional uphill barriers for one of these two reactions, and therefore severely limit overall water splitting efficiency. Since the downward band bending reduces with increase in $\mathrm{T}_{\mathrm{Mg}}$ in the $p$-type surface regime (Fig. 2c, Regime II), efficient carrier transport and therefore high photocatalytic activity is expected for optimized $p$-doping levels.

$\mathbf{R h} / \mathrm{Cr}_{2} \mathrm{O}_{3}$ cocatalyst. To further enhance the charge carrier extraction and provide more active sites for gas generation, $\mathrm{Rh} /$ Chromium-oxide $\left(\mathrm{Cr}_{2} \mathrm{O}_{3}\right)$ cocatalyst nanoparticles are deposited on all the samples (A-F) using photodeposition process from liquid precursors (Methods section) ${ }^{14}$. Figure 3 shows (a) a low magnification STEM secondary electron (SE) image; and (b) a STEM-high-angle annular dark-field (HAADF) image of $\mathrm{Rh} /$ $\mathrm{Cr}_{2} \mathrm{O}_{3}$ nanoparticle-decorated $\mathrm{GaN}$ nanowire. These images reveal that the $\mathrm{Rh} / \mathrm{Cr}_{2} \mathrm{O}_{3}$ nanoparticles are successfully deposited in the form of core-shell nanostructures on GaN nanowire surfaces. In addition, the $\mathrm{Rh}$ core and $\mathrm{GaN}$ nanowire show single crystalline structure. The presence of metallic Rh, mixed Rh-Cr oxide, and $\mathrm{Cr}_{2} \mathrm{O}_{3}$ nanoparticles was further confirmed by ARXPS (Supplementary Note 2 and Supplementary Fig. 6). The Rh core facilitates carrier migration to the nanowire surface and reduces the activation energy required for gas evolution. The $\mathrm{Cr}_{2} \mathrm{O}_{3}$ shell suppresses the back reaction to form $\mathrm{H}_{2} \mathrm{O}$ from $\mathrm{H}_{2}$ and $\mathrm{O}_{2}$ on the $\mathrm{Rh}$ core $^{15}$. The role of cocatalyst was further elucidated by controlexperiments (Supplementary Note 3 and Supplementary Fig. 7). Figure $3 \mathrm{c}$ shows a schematic of the core-shell $\mathrm{Rh} / \mathrm{Cr}_{2} \mathrm{O}_{3}$ nanoparticle deposited $\mathrm{GaN}$ nanowire for overall water splitting, illustrating the reaction mechanism on the nonpolar surfaces of $\mathrm{GaN}$ nanowires.

Overall pure water splitting. The $\mathrm{Rh} / \mathrm{Cr}_{2} \mathrm{O}_{3}$ nanoparticle-decorated $\mathrm{GaN}$ nanowires with different $\mathrm{Mg}$-doping levels were subsequently used for overall pure $(\mathrm{pH} \sim 7.0)$ water splitting under $300 \mathrm{~W}$ full arc Xenon lamp irradiation. Figure 4a shows the $\mathrm{H}_{2}$ evolution rates for different GaN:Mg nanowire samples, which were derived from $6 \mathrm{~h}$ overall water splitting reaction on each sample. It is seen that the $\mathrm{H}_{2}$ evolution rate first shows a marked increase with $\mathrm{Mg}$ cell temperature. Under relatively low doping levels (samples A and B), the photocatalytic activity is low, which is directly correlated to the very large downward surface band bending (Fig. 1d), because of the weakly $n$-type surface-nature as revealed from ARXPS and micro-Raman analysis (Fig. 2c, Regime I, and Fig. 2d). Markedly enhanced overall water splitting performance was measured for sample $\mathrm{D}\left(T_{\mathrm{Mg}}=265^{\circ} \mathrm{C}\right)$, and the $\mathrm{H}_{2}$ evolution rate was nearly $4 \mathrm{~mol} \mathrm{~h}^{-1} \mathrm{~g}^{-1}$, which is more than two orders of magnitude higher, compared with the nominally undoped GaN nanowires ${ }^{14}$. This drastic improvement can be well explained by the $p$-type surfaces with significantly reduced downward band bending (Fig. 2c, Regime II, and Fig. 2d), which may become relatively flat in aqueous solution, thereby enabling balanced and much more efficient redox reactions. For samples $\mathrm{E}\left(T_{\mathrm{Mg}}=280^{\circ} \mathrm{C}\right)$ and $\mathrm{F}\left(T_{\mathrm{Mg}}=300^{\circ} \mathrm{C}\right)$, however, the activity shows a decreasing trend with increase in $\mathrm{Mg}$ cell temperature, which is likely to be related to the degradation of $\mathrm{GaN}$ nanowire crystalline quality at very high $\mathrm{Mg}$-doping level ${ }^{24,34,35}$ (Supplementary Note 4). For optimum Mg-doping concentration (sample D), the apparent quantum efficiency can reach $\sim 18 \%$ (Supplementary Note 5 ). The internal quantum efficiency, as shown in Fig. $4 \mathrm{a}$, is also derived for different $\mathrm{Mg}$ doped samples under $\sim 200-365 \mathrm{~nm}$ wavelength (Supplementary Note 5). It can reach $\sim 51 \%$ for $\mathrm{GaN}$ nanowires with optimum surface band bending (sample D), which is the highest value reported for spontaneous overall water splitting under any broadband light irradiation to our knowledge ${ }^{4,36,37}$.

\section{Discussion}

In order to reveal the stability of the GaN photocatalyst, repeated experiments were performed using the same sample. Figure $4 \mathrm{~b}$ shows the evolution of $\mathrm{H}_{2}$ and $\mathrm{O}_{2}$ as a function of irradiation time for the optimized GaN:Mg (sample D) in pure water. No sacrificial reagents were added in the reaction. The rates of $\mathrm{H}_{2}$ and $\mathrm{O}_{2}$ evolution were $\sim 1,525$ and $\sim 763 \mu \mathrm{molh}^{-1}$, respectively. The $\mathrm{H}_{2} / \mathrm{O}_{2}$ ratio is nearly $2: 1$, indicating a balanced redox reaction of water on the nanowire photocatalyst. Visible bubbles were clearly observed from the sample surface upon irradiation (Supplementary Movie 1). The turnover number, defined as the ratio of the total amount of gas evolved $(50,428 \mu \mathrm{mol})$ to $\mathrm{GaN}$ catalyst $(4.62 \mu \mathrm{mol})$, exceeded $\sim 10,915$ during the course of $\sim 22 \mathrm{~h}$ of reaction. Repeated experiments on the same sample did not show any degradation of the photocatalytic activity, demonstrating excellent stability of the Mg-doped GaN nanowires. The turnover number in terms of the ratio of the rate of $\mathrm{H}_{2}$ molecules formed to the rate of absorbed photons is also plotted as a function of time (Supplementary Fig. 8), further confirming excellent stability of the nanowire photocatalyst. The slight variations in $\mathrm{H}_{2}$ and $\mathrm{O}_{2}$ evolution observed for different cycles are attributed to the variations in alignment between the light source and the sample surface.

Figure 4c shows a SEM image of the nanowire arrays (sample D) after overall water splitting for $\sim 22 \mathrm{~h}$. The nanowires 
a

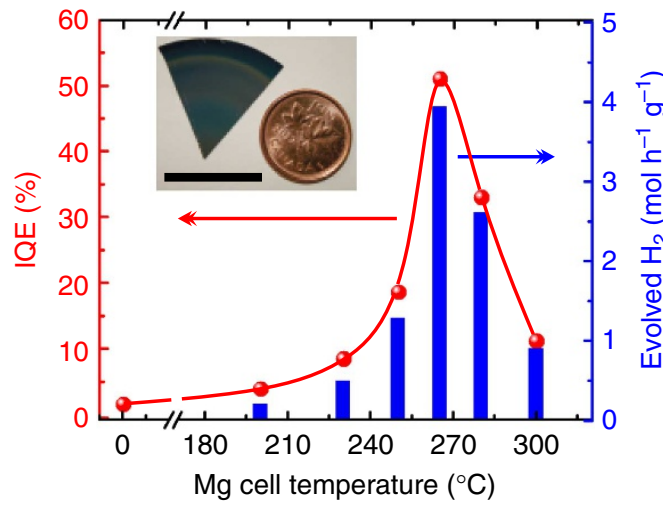

b

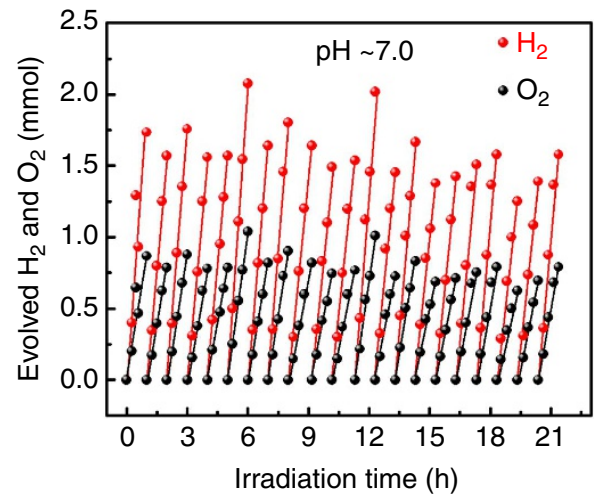

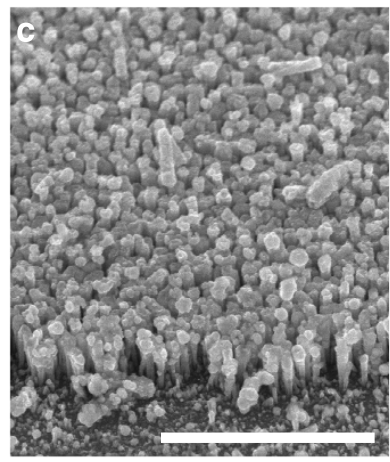
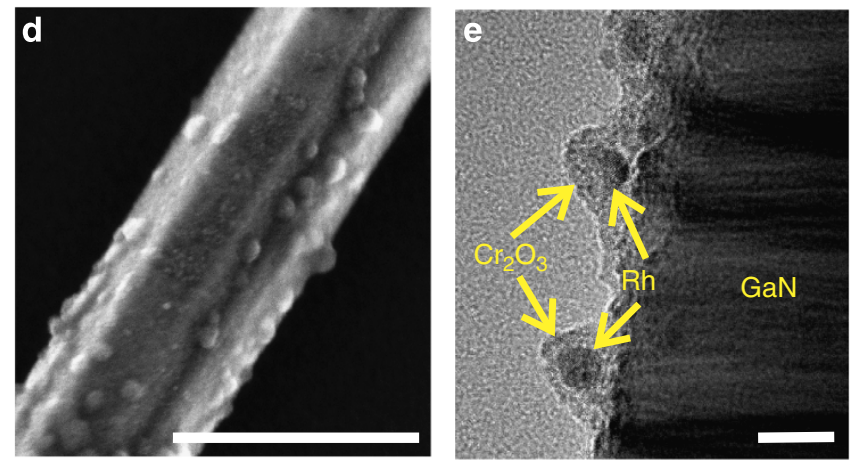

Figure 4 | Overall water splitting and stability of GaN:Mg nanowires. (a) $\mathrm{H}_{2}$ evolution rate and internal quantum efficiency (IQE) for different GaN:Mg samples in overall pure water splitting. Reactions were performed using $\sim 0.387 \mathrm{mg} \mathrm{GaN}$ nanowire catalyst in pure water under illumination of a Xenon lamp $(300 \mathrm{~W})$. The sample size is shown in the inset. Scale bar $2 \mathrm{~cm}$. (b) Repeated cycles of overall pure water splitting for GaN:Mg: $265^{\circ} \mathrm{C}$ (sample D), showing the stability of the nanowires. (c) A bird's-eye view SEM image of the GaN nanowire arrays after $\sim 22 \mathrm{~h}$ of overall water splitting. Scale bar, $2 \mu \mathrm{m}$. (d) Low-magnification STEM-SE image of a single nanowire after overall water splitting reaction for $\sim 22 \mathrm{~h}$. Scale bar, $100 \mathrm{~nm}$. (e) High-resolution BF TEM image after reaction, showing the stability of core-shell $\mathrm{Rh} / \mathrm{Cr}_{2} \mathrm{O}_{3}$ nanoparticles and $\mathrm{GaN}$ nanowire. Scale bar, $10 \mathrm{~nm}$. The solid lines are guide to the eye.

remain stable during the course of the reaction. A lowmagnification STEM-SE image of a single nanowire, as shown in Fig. 4d, further confirms that the cocatalyst nanoparticledecorated GaN nanowire were not etched during the course of reaction, which can be partially attributed to the surface freeholes of $\mathrm{Mg}$-doped $\mathrm{GaN}$ that can prevent the catalyst from photocorrosion caused by oxidation ${ }^{38}$. The core-shell $\mathrm{Rh} / \mathrm{Cr}_{2} \mathrm{O}_{3}$ nanoparticles are also stable in photocatalytic environment, as shown in the BF TEM image in Fig. 4e.

In summary, we have demonstrated that by tuning the surface Fermi level through controlled Mg-dopant incorporation, the quantum efficiency for spontaneous pure water splitting on $\mathrm{GaN}$ nanowire arrays can be enhanced by nearly two orders of magnitude, and the activity can reach $\sim 4.0 \mathrm{~mol}$ hydrogen $\mathrm{h}^{-1} \mathrm{~g}^{-1}$, which is much higher than previously reported photocatalyst materials ${ }^{36}$. Future work includes the demonstration of high efficiency and stable overall water splitting under visible and infrared light irradiation by engineering the surface charge properties of InGaN nanowires.

\section{Methods}

MBE growth. Catalyst-free vertically aligned GaN nanowires were grown on $n$-Si (111) substrate by radio frequency plasma-assisted MBE under nitrogen rich conditions. Thermal effusion cells were used for $\mathrm{Ga}$ and $\mathrm{Mg}$. The native oxide on $\mathrm{Si}$ substrate was etched with $10 \%$ hydrofluoric acid (HF) for $30 \mathrm{~s}$ before loading into the MBE chamber. The growth parameters include a growth temperature of $\sim 750^{\circ} \mathrm{C}$, nitrogen flow rate of 1.0 standard cubic centimeters per minute $(\mathrm{sccm})$, a forward plasma power of $\sim 350 \mathrm{~W}$ and a Ga beam equivalent pressure of $\sim 6 \times 10^{-8}$ Torr. The growth duration was $\sim 4 \mathrm{~h}$. The $\mathrm{Mg}$ beam equivalent pressure was varied from $\sim 0.2 \times 10^{-10}$ to $\sim 1.5 \times 10^{-9}$ Torr for Mg cell temperatures from 200 to $300{ }^{\circ} \mathrm{C}$. Note that, because of the presence of conduction and valence band offsets at the GaN/Si interface, the photoexcited carriers in $\mathrm{Si}$ substrate do not take part in the photochemical reaction ${ }^{15}$.

Transmission electron microscopy. A CM200 microscope with an accelerating voltage of $200 \mathrm{kV}$ was used to obtain BF TEM images. For STEM-SE and STEMHAADF imaging, a Hitachi HD2700 Cs-corrected STEM with a cold-field emission emitter operated at $200 \mathrm{kV}$ and with an electron beam diameter of $\sim 0.1 \mathrm{~nm}$ was used.

PL measurement. A 325-nm He-Cd laser (Kimmon Koha) was used as the excitation source for the PL measurement of the nanowire heterostructure. The PL was spectrally resolved by a high-resolution spectrometer and detected by a photomultiplier tube. Room temperature PL spectrum of GaN:Mg nanowires, with $\mathrm{Mg}$ effusion cell temperature of $230^{\circ} \mathrm{C}$, is shown in Supplementary Fig. 2.

Micro-Raman measurement. Micro-Raman measurements were carried out at room temperature with an external $488 \mathrm{~nm}$ Argon-ion laser through a $\times 100$ objective with a numerical aperture $\sim 0.9$. The focused laser spot size was $\sim 1 \mu \mathrm{m}$ and the estimated incident power was $\sim 40 \mathrm{~mW}$. The Raman signal was collected by a Synapse CCD air-cooled detector. The incident laser was parallel with the hexagonal $c$ axis of the nanowires, and the laser light was scattered without polarization analysis $(z(.)$.$z direction).$

Photocatalytic reaction. The photocatalytic reaction was performed in a Pyrex chamber with a quartz lid. A 300-W Xenon lamp (Cermax, PE300BUV) was used as an outer irradiation source. Distilled water $(\mathrm{pH} \sim 7.0)$ was used for the pure water splitting reaction. The $\mathrm{H}_{2}$ half reaction was performed in the presence of $\mathrm{CH}_{3} \mathrm{OH}\left(20\right.$ vol.\%) as electron donor and $\mathrm{Rh}$ nanoparticles as cocatalyst. For $\mathrm{O}_{2}$ half reaction, $\mathrm{AgNO}_{3}(0.1 \mathrm{M})$ was used as electron acceptor. The water was purged with Ar for $30 \mathrm{~min}$ before each experiment. The chamber was then evacuated. The gases produced from the reaction were sampled using a vacuum tight syringe. 
A gas chromatograph (Shimadzu GC-8A) equipped with thermal conductivity detector and high-purity $\mathrm{Ar}$ carrier gas was used to analyse the reaction-evolved gases. The experimental error in the evolution of $\mathrm{H}_{2}$ and $\mathrm{O}_{2}$ is estimated to be $\sim 10 \%$, which is ascribed to the manual sampling of the gases.

Photodeposition of $\mathbf{R h} / \mathrm{Cr}_{\mathbf{2}} \mathbf{O}_{3}$ core-shell nanoparticles. The deposition of $\mathrm{Rh} /$ $\mathrm{Cr}_{2} \mathrm{O}_{3}$ core-shell nanoparticles on the $\mathrm{GaN}$ nanowires was performed following a two-step photodeposition process. First, $2 \mu \mathrm{l}$ of $0.2 \mathrm{M}$ sodium hexachlororhodate(III) $\left(\mathrm{Na}_{3} \mathrm{RhCl}_{6}\right.$, Sigma-Aldrich), $12 \mathrm{ml}$ of $\mathrm{CH}_{3} \mathrm{OH}$ and $60 \mathrm{ml}$ of distilled water (purged with pure Argon for $30 \mathrm{~min}$ ) were placed in a $460 \mathrm{ml}$ Pyrex chamber (Kimble Chase) with quartz lid. The GaN nanowire photocatalyst (on Si substrate) was placed in the Pyrex chamber using a homemade polytetrafluoroethylene holder. The chamber was then pumped down for $10 \mathrm{~min}$ and subsequently irradiated for 30 min using $300 \mathrm{~W}$ Xenon lamp (Cermax, PE300BUV) for photo-assisted deposition of Rh nanoparticles. The sample was dried in air for a few minutes. In the second step, the $\mathrm{Cr}_{2} \mathrm{O}_{3}$ was deposited following the abovementioned procedure by $2 \mu \mathrm{l}$ of $0.2 \mathrm{M}$ potassium chromate $\left(\mathrm{K}_{2} \mathrm{CrO}_{4}\right.$, SigmaAldrich) as precursor.

Angle-resolved X-ray photoelectron spectroscopy. The monochromatic $\mathrm{Al}-\mathrm{K} \alpha$ $\mathrm{X}$-ray source $(\mathrm{h} v=1486.6 \mathrm{eV})$ was placed $60^{\circ}$ with the surface normal to excite the nonpolar surfaces of GaN nanowire arrays. The high-resolution XPS spectra were obtained using an X-ray beam size of $400 \mu \mathrm{m}$, pass energy of $50 \mathrm{eV}$ and a step size of $0.1 \mathrm{eV}$. Individual peak fitting was performed using convolution of Lorentzian and Gaussian line shapes ( $\mathrm{L} / \mathrm{G}=30 \%$ ) with Avantage software (Thermo Scientific). The binding energies were calibrated with both $\mathrm{Au}-4 \mathrm{f}$ peak $(84.0 \mathrm{eV})$ and $\mathrm{C}-1 \mathrm{~s}$ peak $(285.0 \mathrm{eV})$ before each experiment. The $E_{\mathrm{F}}-E_{\mathrm{V}}$ was estimated from ARXPS valence spectrum with linear extrapolation of the valence band leading edge and the baseline as shown in the inset of Fig. 2c. The intersection between these two lines indicates the position of surface valence band with respect to the surface Fermi level (binding energy $=0 \mathrm{eV})^{27}$.

\section{References}

1. Tachibana, Y., Vayssieres, L. \& Durrant, J. R. Artificial photosynthesis for solar water-splitting. Nat. Photon. 6, 511-518 (2012).

2. Liu, C., Dasgupta, N. P. \& Yang, P. Semiconductor nanowires for artificial photosynthesis. Chem. Mater. 26, 415-422 (2014).

3. Khaselev, O. \& Turner, J. A. A Monolithic photovoltaic-photoelectrochemical device for hydrogen production via water splitting. Science 280, 425-427 (1998).

4. Kudo, A. \& Miseki, Y. Heterogeneous photocatalyst materials for water splitting. Chem. Soc. Rev. 38, 253-278 (2009).

5. Maeda, K. et al. Photocatalyst releasing hydrogen from water. Nature 440, 295-295 (2006).

6. Reece, S. Y. et al. Wireless solar water splitting using silicon-based semiconductors and earth-abundant catalysts. Science 334, 645-648 (2011).

7. Walter, M. G. et al. Solar water splitting cells. Chem. Rev. 110, 6446-6473 (2010).

8. Liu, C., Tang, J., Chen, H. M., Liu, B. \& Yang, P. A fully integrated nanosystem of semiconductor nanowires for direct solar water splitting. Nano Lett. 13, 2989-2992 (2013)

9. Tong, H. et al. Nano-photocatalytic materials: possibilities and challenges. $A d v$. Mater. 24, 229-251 (2012).

10. Wang, G. et al. Hydrogen-treated $\mathrm{TiO}_{2}$ nanowire arrays for photoelectrochemical water splitting. Nano Lett. 11, 3026-3033 (2011).

11. Khanal, D. R., Walukiewicz, W., Grandal, J., Calleja, E. \& Wu, J. Determining surface Fermi level pinning position of InN nanowires using electrolyte gating. Appl. Phys. Lett. 95, 173114 (2009).

12. Walle, C. G. V. d. \& Segev, D. Microscopic origins of surface states on nitride surfaces. J. Appl. Phys. 101, 081704 (2007).

13. Nozik, A. J. \& Memming, R. Physical chemistry of semiconductor - liquid interfaces. J. Phys. Chem. 100, 13061-13078 (1996).

14. Wang, D. et al. Wafer-level photocatalytic water splitting on GaN nanowire arrays grown by molecular beam epitaxy. Nano Lett. 11, 2353-2357 (2011).

15. Kibria, M. G. et al. One-step overall water splitting under visible light using multiband InGaN/GaN nanowire heterostructures. ACS Nano 7, 7886-7893 (2013).

16. Moses, P. G. \& Walle, C. G. V. d. Band bowing and band alignment in InGaN alloys. Appl. Phys. Lett. 96, 021908 (2010).

17. Bertelli, M. et al. Atomic and electronic structure of the nonpolar GaN(1100) surface. Phys. Rev. B 80, 115324 (2009).

18. Ivanova, L. et al. Surface states and origin of the Fermi level pinning on nonpolar GaN(11̄00) surfaces. Appl. Phys. Lett. 93, 192110 (2008).

19. Wang, J., Pedroza, L. S., Poissier, A. \& Fernández-Serra, M. V. Water dissociation at the $\mathrm{GaN}(10 \overline{1} 0)$ surface: structure, dynamics and surface acidity. J. Phys. Chem. C 116, 14382-14389 (2012).

20. Shen, X. et al. Photocatalytic water oxidation at the GaN (1010) - water interface. J. Phys. Chem. C 114, 13695-13704 (2010).

21. Barbet, S. et al. Surface potential of n- and p-type GaN measured by Kelvin force microscopy. Appl. Phys. Lett. 93, 212107 (2008).
22. Sezen, H., Ozbay, E., Aktas, O. \& Suzer, S. Transient surface photovoltage in $\mathrm{n}$ - and $\mathrm{p}-\mathrm{GaN}$ as probed by $\mathrm{x}$-ray photoelectron spectroscopy. Appl. Phys. Lett. 98, 111901 (2011).

23. Fàbrega, C. et al. Tuning the Fermi level and the kinetics of surface states of $\mathrm{TiO}_{2}$ nanorods by means of ammonia treatments. J. Phys. Chem. C 117, 20517-20524 (2013).

24. Stoica, T. \& Calarco, R. Doping of III-nitride nanowires grown by molecular beam epitaxy. IEEE J. Sel. Top. Quantum Electron. 17, 859-868 (2011).

25. Cimpoiasu, E. et al. The effect of $\mathrm{Mg}$ doping on $\mathrm{GaN}$ nanowires. Nanotechnology 17, 5735 (2006).

26. Arbiol, J. et al. Triple-twin domains in $\mathrm{Mg}$ doped $\mathrm{GaN}$ wurtzite nanowires: structural and electronic properties of this zinc-blende-like stacking. Nanotechnology 20, 145704 (2009).

27. Chambers, S. A., Droubay, T., Kaspar, T. C. \& Gutowski, M. Experimental determination of valence band maxima for $\mathrm{SrTiO}_{3}, \mathrm{TiO}_{2}$, and $\mathrm{SrO}$ and the associated valence band offsets with $\mathrm{Si}(001)$. J. Vac. Sci. Technol. B Microelectron. Nanometer Struct. 22, 2205-2215 (2004).

28. Wu, C. I., Kahn, A., Taskar, N., Dorman, D. \& Gallagher, D. GaN (0001)- $(1 \times 1)$ surfaces: composition and electronic properties. J. Appl. Phys. 83, 4249-4252 (1998).

29. Zhong, Z., Qian, F., Wang, D. \& Lieber, C. M. Synthesis of p-type gallium nitride nanowires for electronic and photonic nanodevices. Nano Lett. 3, 343-346 (2003).

30. Kirste, R. et al. Compensation effects in GaN:Mg probed by Raman spectroscopy and photoluminescence measurements. J. Appl. Phys. 113, 103504 (2013)

31. Hashizume, $\mathrm{T}$. Effects of $\mathrm{Mg}$ accumulation on chemical and electronic properties of Mg-doped p-type GaN surface. J. Appl. Phys. 94, 431-436 (2003).

32. Jeganathan, K. et al. Raman scattering of phonon-plasmon coupled modes in self-assembled GaN nanowires. J. Appl. Phys. 105, 123707 (2009).

33. Hiroshi, H. Properties of GaN and related compounds studied by means of Raman scattering. J. Phys: Condens. Matter 14, R967 (2002).

34. Furtmayr, F. et al. Optical properties of Si- and Mg-doped gallium nitride nanowires grown by plasma-assisted molecular beam epitaxy. J. Appl. Phys. 104, 074309 (2008).

35. Furtmayr, F. et al. Nucleation and growth of GaN nanorods on $\mathrm{Si}$ (111) surfaces by plasma-assisted molecular beam epitaxy-The influence of Si- and Mg-doping. J. Appl. Phys. 104, 034309 (2008).

36. Chen, X., Shen, S., Guo, L. \& Mao, S. S. Semiconductor-based photocatalytic hydrogen generation. Chem. Rev. 110, 6503-6570 (2010).

37. Maeda, K. Photocatalytic water splitting using semiconductor particles: history and recent developments. J. Photochem. Photobiol. C Photochem. Rev. 12, 237-268 (2011).

38. Aryal, K., Pantha, B. N., Li, J., Lin, J. Y. \& Jiang, H. X. Hydrogen generation by solar water splitting using $\mathrm{p}-\mathrm{InGaN}$ photoelectrochemical cells. Appl. Phys. Lett. 96, 052110 (2010).

\section{Acknowledgements}

This work was supported by the Natural Sciences and Engineering Research Council of Canada (NSERC). Part of the work was performed in the Micro-fabrication Facility at McGill University. Electron microscopy images and analysis were carried out at IREQ of Hydro-Québec and Facility for Electron Microscopy Research (FEMR), McGill University. The ARXPS and micro-Raman analysis were performed at McGill Institute for Advanced Materials (MIAM). We would like to acknowledge Professor M.P. Andrews and $\mathrm{Mr}$ T. Gonzales at McGill University for their help with micro-Raman measurements, and Dr J. Lefebvre at Université de Montréal for her assistance in ARXPS data analysis.

\section{Author contributions}

M.G.K. and Z.M. designed the study; M.G.K. and F.A.C. conducted the photocatalytic experiments; M.G.K. and S.Z. performed scanning electron microscopy, photoluminescence and X-ray photoelectron spectroscopy measurements; Q.W. conducted micro-Raman measurement and analysis; S.Z., H.P.T.N. and Z.M. conducted MBE growth of GaN nanowires; M.L.T. contributed to the transmission electron microscopy analysis; M.G.K., Z.M. and H.G. contributed to the result analysis and discussions; and F.A.C. and M.G.K. contributed to the quantum efficiency calculation. The paper was written by M.G.K. and Z.M. with contributions from other coauthors.

\section{Additional information}

Supplementary Information accompanies this paper at http://www.nature.com/ naturecommunications

Competing financial interests: The authors declare no competing financial interests

Reprints and permission information is available online at http://npg.nature.com/ reprintsandpermissions/

How to cite this article: Kibria, M. G. et al. Tuning the surface Fermi level on $p$-type gallium nitride nanowires for efficient overall water splitting. Nat. Commun. 5:3825 doi: $10.1038 /$ ncomms4825 (2014). 